\title{
Linkage disequilibrium reveals different demographic history in egg laying chickens
}

\author{
Saber Qanbari ${ }^{1 *}$, Maria Hansen ${ }^{1}$, Steffen Weigend ${ }^{2}$, Rudolf Preisinger $^{3}$, Henner Simianer $^{1}$
}

\begin{abstract}
Background: The availability of larger-scale SNP data sets in the chicken genome allows to achieve a higher resolution of the pattern of linkage disequilibrium (LD). In this study, $36 \mathrm{k}$ and $57 \mathrm{k}$ genotypes from two independent genotyping chips were used to systematically characterize genome-wide extent and structure of LD in the genome of four chicken populations. In total, we analyzed genotypes of 454 animals from two commercial and two experimental populations of white and brown layers which allows to some extent a generalization of the results.

Results: The number of usable SNPs in this study was $19 \mathrm{k}$ to $37 \mathrm{k}$ in brown layers and $8 \mathrm{k}$ to $19 \mathrm{k}$ in white layers. Our analyzes showed a large difference of LD between the lines of white and brown layers. A mean value of $r^{2}=$ $0.73 \pm 0.36$ was observed in pair-wise distances of $<25 \mathrm{~Kb}$ for commercial white layers, and it dropped to $0.60 \pm$ 0.38 with distances of 75 to $120 \mathrm{~Kb}$, the interval which includes the average inter-marker space in this line. In contrast, an overall mean value of $r^{2}=0.32 \pm 0.33$ was observed for SNPs less than $25 \mathrm{~Kb}$ apart from each other and dropped to $0.21 \pm 0.26$ at a distance of $100 \mathrm{~kb}$ in commercial brown layers. There was a remarkable similarity of the LD patterns among the two populations of white layers. The same was true for the two populations of brown layers, while the LD pattern between white and brown layers was clearly different. Inferring the population demographic history from LD data resulted in a larger effective population size in brown than white populations, reflecting less inbreeding among brown compared to white egg layers.
\end{abstract}

Conclusions: We report comprehensive LD map statistics for the genome of egg laying chickens with an up to 3 times higher resolution compared to the maps available so far. The results were found to be consistent between analyzes based on the parallel SNP chips and across different populations (commercial vs. experimental) within the brown and the white layers. It is concluded that the current density of usable markers in this study is sufficient for association mapping and the implementation of genomic selection in these populations to achieve a similar accuracy as in implementations of association mapping and genomic selection in mammalian farm animals.

\section{Background}

Linkage disequilibrium (LD), the non-random association of alleles at two or more loci, has been in the focus of much attention recently, because of its usefulness in determining the actual genes responsible for variation of economically important traits through association mapping in livestock populations [1,2]. Information on the extent of LD in genomic regions harboring quantitative trait loci (QTLs) is necessary for effective applications of marker assisted selection in commercial breeding

\footnotetext{
* Correspondence: sqanbar@gwdg.de

1 Animal Breeding and Genetics Group, Department of Animal Sciences,

Georg-August University, 37075 Göttingen, Germany

Full list of author information is available at the end of the article
}

programs [3]. Another appealing application of LD information is inferring population demography based on the changes in the historical effective population size $(\mathrm{Ne})$. Theoretical analyzes based on the well-known formula suggested by Sved [4] allow assessing the development of historical effective population size by comparing the decay of LD over intervals of increasing map distance. However, additional factors such as genetic drift, selection within populations, and population admixture can also cause LD between marker pairs or markers and traits, and these mechanisms might affect non-syntenic loci across chromosomes, i.e. in the absence of physical linkage. Therefore, information on the local magnitude of LD and a detailed profile of the
C Biomed Central

C 2010 Qanbari et al; licensee BioMed Central Ltd. This is an Open Access article distributed under the terms of the Creative Commons Attribution License (http://creativecommons.org/licenses/by/2.0), which permits unrestricted use, distribution, and reproduction in any medium, provided the original work is properly cited. 
recombination variability and blocking structure is of key importance for the genome-based analysis of population history and for the fine-tuning of applications like association mapping and genomic selection.

Since the first genetic linkage map of chicken was published by Serebrovsky and Petrov [5] several versions of linkage maps, mostly based on microsatellite markers, have been constructed [6-8]. These studies have reported substantial LD over long distances for pairs of loci being up to $5 \mathrm{cM}$ apart. As in many other species, microsatellites recently have been replaced by single nucleotide polymorphisms (SNPs) and maps based on high-density SNP arrays reveal much lower levels of LD, limited to $\leq 100 \mathrm{~kb}$ [9-11]. The latest map included 13,340 markers with a total map length of 3,054 cM in broilers [12].

The availability of large-scale SNP data sets in the chicken genome [13] allowed to increase the marker density and to achieve a comprehensive coverage of the chicken genome. Benefiting from recently established Illumina $60 \mathrm{~K}$ genotyping chips for the chicken genome, in this study, we (1) construct a new LD map with higher resolution, (2) compare the extent and structure of LD and haplotype blocks between lines of brown and white egg laying chickens, and (3) use LD statistics to estimate and compare present and historic effective population sizes of the studied populations which have a diverse historical background. Since these analyzes are based on the genotypes available from two independent SNP chips and two independent brown and white layer lines, respectively, the results can be validated across genotyping technologies and populations.

\section{Methods}

\section{Populations studied}

This study is based on two experimental (E) and two commercial $(\mathrm{C})$ populations, each comprising a white (W) and a brown (B) layer breed (Table 1). We henceforth use the code WE for the experimental white layer line, and accordingly BE, WC, and BC. Experimental and commercial lines with systematical differences in breeding history, sample size, and the SNP chip were used for genotyping. This structure allows to some extent a generalization of results.

Table 1 summarizes information concerning population, sample size, status and the main breeding purpose and scheme of the 454 birds composing two data sets of this study.

Data set I comprised two experimental pure bred chicken lines, WE and BE representing White Leghorn and New Hampshire chickens, respectively. The selected lines have been maintained at the Institute of Farm Animal Genetics (FLI) Neustadt, Mariensee. Hatching eggs for White Leghorn chicken were imported to former Institute of Small Animal Breeding in Celle in 1965 from the Cornell Line $\mathrm{K}$ selected for resistance to neoplasm. The sub-line WE had been established with the aim of selecting for susceptibility for ALV A/B infection. $\mathrm{BE}$ is an experimental brown layer chicken line founded in 1970 in the former German Democratic Republic (VEG Vogelsang). Each population was composed of 10 sire families made up by 1 sire and 10 dams per family. After each generation roosters were systematically rotated among families (e.g; a rooster from first family artificially inseminates second family and so on) with no selection and a generation interval of 1 year (for more information see [14]).

Twenty five birds of each experimental line were sampled from two consecutive years (2003 and 2004), reflecting two subsequent generations. In each line the sire of each family (e.g., 10 sires per line) and one or two females per family $(n=15)$, respectively, were sampled to represent the complete variation of the lines.

Data set II was composed of two commercial egg laying strains of the Lohmann Tierzucht GmbH. The first population BC comprised 204 laying hens produced from a two way cross of Lohmann Brown lines. The pure lines have their origins in Rhode Island Red and White Rock, respectively, and are closed populations which are selected for all economically important traits under pure and crossbred conditions using genetic evaluations based on multiple trait best linear unbiased prediction. The genotyped BC individuals are F1 animals of a cross of pure breeds. This means, that after haplotyping we

Table 1 Description of the genotyped birds

\begin{tabular}{|c|c|c|c|c|c|c|}
\hline Dataset & Line & Breed & Purpose & Animals (n) & $\begin{array}{l}\text { Breeding } \\
\text { scheme }\end{array}$ & Pedigree \\
\hline 1 & WE & White Leghorn & experimental & 25 & $\begin{array}{l}\text { conservation breeding using } \\
\text { rooster rotation }\end{array}$ & 2000-2007 \\
\hline । & $\mathrm{BE}$ & New Hampshire & experimental & 25 & & \\
\hline$\|$ & WC & White Leghorn & commercial & 200 & commercial selection programme & 1992-2008 \\
\hline \multirow[t]{2}{*}{$\|$} & $\mathrm{BC}$ & Rhode Island Red and White Rock & commercial & 204 & & \\
\hline & & & & $\sum 454$ & & \\
\hline
\end{tabular}


observe in one individual for each autosome a combination of two chromosomes originating from either of the two parental breeds, respectively, without being able to assign haplotypes to the breed of origin. Note, however, that no recombination across haplotypes of the two different parental lines was possible and hence the observed LD was not generated by population admixture. The parameters estimated from these genotypes thus reflect a mixture of two purebred brown layer populations, rather than a single population.

The second commercial population WC consisted of 200 White Leghorn females from a pure line. The samples comprised were half sib groups. It is generally recommended to use maternal haplotypes for evaluating the extent and pattern of LD, because LD from paternal haplotypes may reflect LD within sire families, rather than in the wider population. However, these relationships are not expected to bias estimates of LD, because the small size of half sib families limits genetic contribution of each sire in the relatively large sample taken from this line [9]. Sampling from both commercial lines was done in 2008.

\section{SNP genotypes and data preparation}

DNA was extracted from fresh blood samples using standard DNA isolation procedures [15]. SNP genotyping for data set I was done by DNA LandMarks Inc., Quebec, Canada, using publicly available chicken $60 \mathrm{~K}$ chips produced by Illumina Inc. for the genome-wide marker-assisted selection (GWMAS) Consortium. The total number of SNPs and the mean distance between adjacent markers in this chip were 57'635 and $17.91 \mathrm{~kb}$, respectively.

Data set II was genotyped with a new chicken genotyping BeadChip which was ordered exclusively by Lohmann Tierzucht $\mathrm{GmbH}$ and established in parallel by Illumina Inc. The Lohmann chip contains a total of 36'455 SNPs with a mean neighbor marker distance of $29.82 \mathrm{~kb}$.

For the purposes of this study, only autosomal SNPs were included in the LD analysis. To ensure the highest possible data quality, a series of filters was employed to remove lower quality markers and insecure genotypes of individuals. We eliminated samples with $\geq 5 \%$ missing genotypes and SNPs which were assigned to unmapped contigs or were not positioned according to the latest reference assembly of the chicken genome (Build 2.1). Genotypes were discarded if they had quality scores < $95 \%$. We also restricted the analysis to SNPs showing a minor allele frequency (MAF) of at least $5 \%$ after filtering. SNPs not matching this criterion were excluded for two reasons: it has been shown that SNPs with low frequency have little power for the detection of LD [16,17]. Furthermore, SNPs with lower allele frequencies increase the number of lower-frequency haplotypes, and the inclusion of rare population-specific SNPs leads to the addition of population-specific haplotypes [18]. The number of heterozygous loci was determined and used to estimate the average heterozygosity for all individuals. Furthermore, MAF and observed heterozygosity were determined for each SNP.

\section{Haplotype inference and block partitioning}

Haplotypes generally have more information content than individual SNPs in genome-wide studies, and they provide valuable information on the evolutionary history of a population. In this study, the inference of haplotype pairs as well as the imputation of missing genotypes was carried out directly on the basis of unphased genotype data for each chromosome within each population using the EM algorithm implemented in fastPHASE [19].

Genomic haplotypes can be partitioned into discrete blocks in such a way that haplotype diversity is constrained within each block and is high between the blocks. We used HAPLOVIEW v4.1 [20] to determine haplotype block boundaries and to estimate within-block haplotype diversity. The algorithm suggested by Gabriel et al. [21] was used to determine the blocking structure by defining a pair of SNPs to be in "strong LD" if the upper 95\% confidence bound of D' is between 0.7 and 0.98 .

\section{Measure of LD}

Several statistics have been used to measure the LD between a pair of loci. We used $r^{2}$ which is generally accepted as a robust LD parameter [22-24] with direct relevance for implementations related to association mapping and genomic breeding value estimation.

Consider 2 loci, $\mathrm{A}$ and $\mathrm{B}$, each locus having 2 alleles (denoted $\mathrm{A}_{1}, \mathrm{~A}_{2} ; \mathrm{B}_{1}, \mathrm{~B}_{2}$, respectively). We denote $f_{11}, f_{12}$, $f_{21}$, and $f_{22}$ as the frequencies of the haplotypes $\mathrm{A}_{1} \mathrm{~B}_{1}$, $\mathrm{A}_{1} \mathrm{~B}_{2}, \mathrm{~A}_{2} \mathrm{~B}_{1}$, and $\mathrm{A}_{2} \mathrm{~B}_{2}$, respectively; $f_{A 1}, f_{A 2} f_{B 1}$, and $f_{B 2}$ are the frequencies of $A_{1}, A_{2}, B_{1}$, and $B_{2}$, respectively. Following Hill and Weir [25],

$$
r^{2}=\frac{\left(f_{11} f_{22}-f_{12} f_{21}\right)^{2}}{f_{A 1} f_{A 2} f_{B 1} f_{B 2}}
$$

\section{Estimation of historical effective population size}

$N_{e}$ can be estimated from LD data and the availability of dense markers has made this option feasible. For autosomal loci assuming a linear population growth, the expected $r^{2}$ between neutral markers can be related to genetic effective population size $\mathrm{Ne}$ and genetic distance $c$ (in Morgan units) according to the formula

$$
E\left(r^{2}=\frac{1}{\kappa+4 N e \times c}+\frac{1}{n}\right)
$$


where $k=1(\approx 2)$ if mutation is (not) taken into account $[4,26]$ and $n$ is the chromosomal sample size. Simulation studies revealed that estimates of past effective population sizes are not greatly affected by departure from the assumption of a linear population growth [27]. Therefore, without considering mutation $(\kappa=1)$ in the model, the effective population size $N e$, in $\frac{1}{2 c}$ generations ago, can then be estimated from observed $r^{2}$ values related to a given genetic distance $c[27,28]$.

While in most mammals the variability of the average ratio of genetic vs. physical distance across chromosomes is small [29,30], an approximately eightfold $(2.5$ to $21 \mathrm{cM} / \mathrm{Mb}$ ) variation in recombination rate was found among chicken chromosomes [31], with a much higher recombination rate per centiMorgan on the microchromosomes compared to the macrochromosomes. To account for this fact we calculated for a marker interval of physical length $x_{i}$ (in kilobasepairs) on chromosome $i$ the corresponding genetic length of the interval to be $c_{i}=\bar{\rho}_{i} x_{i}$, where $\bar{\rho}_{i}$ is the average ratio of Morgan per kilobasepair on chromosome $i$. The values of $\bar{\rho}_{i}$ were taken from the genetic and physical lengths of chromosomes as reported by ICGSC [31]. The decay of $\mathrm{Ne}$ was then analyzed for each population using LD values averaged in bins of linkage distance and inferring the changes in effective population size from nearly 800 generations ago.

\section{Results}

Marker statistics

Table 2 presents a descriptive summary of SNP numbers and frequencies, genome coverage and inter-marker distance for each population. As shown a substantial proportion of the genetic variation represented by either of the SNP chips used is not displayed in any of the four selected lines. Clearly, the magnitude of the fixation is greater in white layers (58 and $62 \%$ in WE and WC, respectively) compared to the brown layers (27 and 32\% in BE and BC, respectively). Line BE with 37'075 SNPs in use having $27.8 \mathrm{~Kb}$ inter-marker distance and $\mathrm{WC}$ with 8'447 SNPs in use and $112.3 \mathrm{~Kb}$ average adjacent marker spacing showed the densest and sparsest marker panels in this study.

Figure 1 displays the distribution of MAF in the populations studied. Nearly $70 \%$ of SNPs in four populations had MAF larger than 0.2, implying that the effect of low MAF on the overall LD estimates should be small. The almost uniform distribution across frequency classes is due to the ascertainment bias, as discussed by Muir et al. [32] and presumably can be explained by the optimization of SNP arrays with respect to a uniform SNP spacing and MAF distribution. The heterozygosity on average was estimated higher for the brown vs. the white layers and for the commercial vs. the experimental populations. Note that observed heterozygosity is excessive in line $\mathrm{BC}$ because the genotyped animals are $\mathrm{F} 1$ crosses of two pure lines; hence the degree of heterozygosity is partly reflecting the genetic distance between those lines.

\section{Comparison of haploblock structure}

Table 3 and Figure 2 present the statistics of the genome wide haploblock distribution across the populations studied. On average about $80 \%$ of the markers formed haplotypic tracts in WL, while this ratio was between $40 \%$ and $50 \%$ for BL. While line WC had 741 blocks spanning over $50 \%$ of the genome, line BE had 2562 blocks covering only $35 \%$ of the genome, which reflected

Table 2 Characteristics of marker panels used in different populations

\begin{tabular}{|c|c|c|c|c|}
\hline & \multicolumn{2}{|c|}{ Dataset I } & \multicolumn{2}{|c|}{ Dataset II } \\
\hline & BE & WE & $\mathrm{BC}$ & WC \\
\hline Total Number of SNPs & $57^{\prime} 635$ & $57^{\prime} 635$ & $36^{\prime} 455$ & $36^{\prime} 455$ \\
\hline \# SNPs monomorph & $15^{\prime} 675$ & $33^{\prime} 915$ & $11^{\prime} 800$ & $222^{\prime} 815$ \\
\hline \# SNPs ungenotyped & 836 & 894 & 982 & 1'059 \\
\hline \# SNPS with MAF < 5\% & $2^{\prime} 893$ & 1'883 & 2'392 & 450 \\
\hline Genome coverage $^{1}(\mathrm{Mb})$ & 1031 & 1027 & 956 & 949 \\
\hline \# SNPs in use & $37^{\prime} 075$ & $19^{\prime} 802$ & $19^{\prime} 892$ & $8^{\prime} 447$ \\
\hline \# common SNPs ${ }^{2}$ & \multicolumn{2}{|c|}{14653} & \multicolumn{2}{|c|}{6072} \\
\hline Mean adjacent marker spacing (kb) & 27.8 & 51.8 & 48.0 & 112.3 \\
\hline Mean MAF & $0.28 \pm 0.13$ & $0.34 \pm 0.14$ & $0.28 \pm 0.21$ & $0.24 \pm 0.15$ \\
\hline Mean observed heterozygosity & $0.35 \pm 0.15$ & $0.34 \pm 0.15$ & $0.47 \pm 0.21$ & $0.37 \pm 0.13$ \\
\hline Mean expected heterozygosity & $0.37 \pm 0.12$ & $0.36 \pm 0.12$ & $0.37^{3} \pm 0.12$ & $0.36 \pm 0.12$ \\
\hline
\end{tabular}

${ }^{1}$ Genome coverage after filtering

${ }^{2}$ Number of common SNPs in use between BE and WE, and between BC and WC

${ }^{3}$ Expected heterozygosity under the assumption that $B C$ was a pure line, which is not the case 


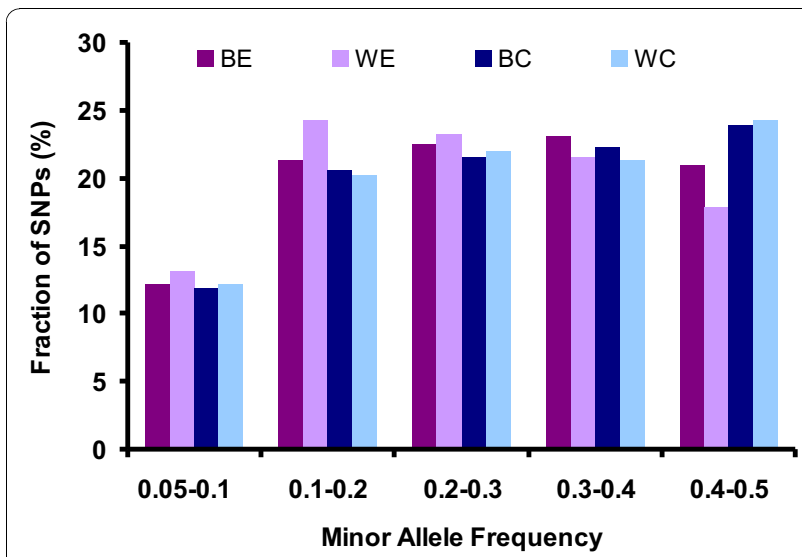

Figure 1 Distribution of minor allele frequency of SNPs in populations.

the longest and shortest blocking structures in the genome, repectively. The mean number of SNPs in the haplotypic tracts ranged from 4.4 to 9.7 among populations with a maximum of 83 SNPs assembling a block in WE.

\section{Comparison of the extent of LD across the genome}

We examined the extent of LD in each line separately. Table 4 summarizes the LD as a function of genetic distance for the lines studied. Analysis of LD showed a large difference between lines representing white and brown layers in both data sets. A mean value of $r^{2}=$ $0.73 \pm 0.36$ was observed in pair-wise distances of $<25$ $\mathrm{Kb}$ for $\mathrm{WC}$ which dropped to $r^{2}=0.60 \pm 0.38$ at distances from 75 to $120 \mathrm{~Kb}$, the interval which includes the average inter-marker space in this line. In line BC an overall mean value of $r^{2}=0.32 \pm 0.33$ was observed for SNPs less than $25 \mathrm{~Kb}$ apart from each other, which dropped to $0.21 \pm 0.26$ at distances from 75 to $120 \mathrm{~Kb}$. There was a remarkable similarity of the LD patterns in two populations of WL from data sets I and II.

In order to visualize the decay of LD we stacked $r^{2}$ and plotted them as a function of inter-marker distance categories $(<0.025,0.025-0.05,0.05-0.075,0.075-0.12$, $0.12-0.2,0.2-0.5,0.5-1.5,1.5-3,3-5$ and 5-10 (Mbp)
(Figure 3). This genome-wide bar plot illustrates the rate at which LD decays with physical distance and forms the basis for comparison between studies. The decay of LD showed a clear exponential trend with physical distance which is typically found in all other data sets and agrees with previous results $[[8,32,33]$ and [11]] and with theory [4].

The threshold for LD being useful for association studies was chosen to be 0.3 in accordance with other studies $[34,23]$. The average proportion of SNPs in useful LD for White and Brown layer lines for the distance of $<25 \mathrm{~Kb}$. was $77.5 \%$ and $42.5 \%$, respectively, This proportion drops to $61 \%$ and $26.3 \%$, respectively, for SNPs $100 \mathrm{~Kb}$ apart from each other. These results showed that the useful LD extended over 5 and $2 \mathrm{Mbp}$ in White and Brown layers, respectively, so that the proportion of SNP pairs in useful LD is above $5 \%$.

To get insight into the significance of LD in different bins of inter-marker distance, we tested the level of departure from expected haplotype frequencies under linkage equilibrium (LE) between markers up to $20 \mathrm{Mb}$ apart using a $\chi^{2}$-test (Foulkes, 2009). The proportion of SNP pairs deviating significantly $(\mathrm{P} \leq 0.05)$ from LE was then computed and compared between micro- and macrochromosomes within commercial populations (Figure 4). As expected, a considerable difference in proportion of SNP pairs in significant LD was observed between brown and white layers. In white layers $27 \%$ of marker pairs showed significant departures at distances up to $20 \mathrm{Mb}$, while this proportion dropped to $10 \%$ in brown layers. A substantial difference was also observed between macro-versus microchromosome which can be attributed to the much higher recombination rates on short chromosomes. It should be noted, that for intervals of 3 to $5 \mathrm{Mb}$ between 30 and 70\% of SNP pairs are in significant LD, despite the fact that the average $r^{2}$ is only between 0.02 and 0.08 (Table 2).

\section{Genome-wide variation in LD}

The extreme heterogeneity of chromosome size is a specific feature of avian genomes such that the genome is

Table 3 A summary statistics of haploblock structure across the populations

\begin{tabular}{|c|c|c|c|c|}
\hline & \multicolumn{2}{|c|}{ Dataset I } & \multicolumn{2}{|c|}{ Dataset II } \\
\hline & BE & WE & BC & WC \\
\hline Blocks (n) & 2562 & 1519 & 2088 & 741 \\
\hline Genome Coverage (Mbp) & 373.7 & 599.4 & 337.1 & 592.0 \\
\hline Mean Block Length (kb) & $145.8 \pm 280.9$ & $394.6 \pm 605$ & $161.4 \pm 324.4$ & $798.9 \pm 1001.6$ \\
\hline BSNPS $^{1}(\%)$ & 41.8 & 74.3 & 46.6 & 85.3 \\
\hline Mean nBSNPs ${ }^{2}$ & $6.1 \pm 6.00$ & $9.7 \pm 9.3$ & $4.4 \pm 3.7$ & $9.7 \pm 8.6$ \\
\hline Max nBSNPs ${ }^{3}$ & 70 & 83 & 69 & 57 \\
\hline
\end{tabular}

1 The total frequency of SNPs forming haploblocks

2,3 the mean and maximum number of SNPs forming haploblocks, respectively 


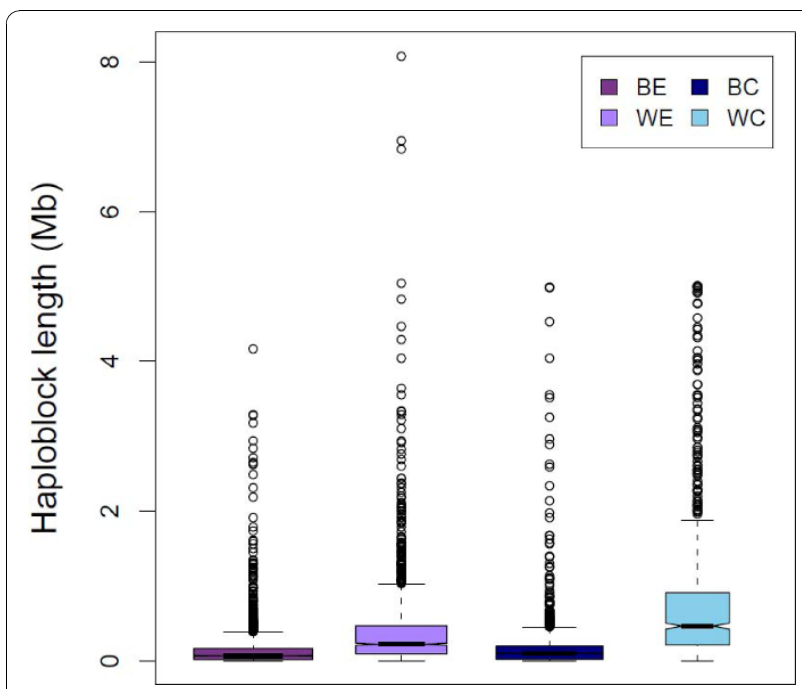

Figure 2 Box plot of haploblock size in different populations.

organized into a few very large chromosomes and many very small chromosomes with substantial structural differences between them. However, there is not a unique classification for chicken chromosomes size. Some reports have classified them as 8 pairs of macrochromosomes, one pair of sex chromosomes, with the remaining 32 pairs being intermediate or microchromosomes [35]. Other arrangements such as the one used by the International Chicken Genome Sequencing Consortium include five pairs of macrochromosomes, five pairs of intermediate chromosomes, and twenty-eight pairs of microchromosomes $[7,36]$. Recombination rates have been found to differ between micro- and macrochromosomes in chickens. This variability first was identified in the chicken genome reporting 2.8-6.4 cM/Mb [31]. For illustration we considered the extent of LD, represented by the average $r^{2}$ for a given physical segment length across chromosomes. Figure 5 shows this statistic $\left(r^{2}\right.$ for an interval size of up to $25 \mathrm{~Kb}$ ) as a function of the physical chromosome length. We fitted a logarithmic function, giving an acceptable fit with an $R^{2}$ value of 0.52 . It should be noted that the often used classification in macro- and microchromosomes [31] does not hold well under this perspective, since the functional relationship between chromosome length and extent of LD is continuous rather than di- or trichotomous.

\section{Inferring population demographic history from LD}

Former reports have shown ensuing reduction in effective population size for commercial breeding populations caused by the intensive artificial selection for many generations [[33,9] and [11]]. In this study we fitted Sved's [4] equation to the $r^{2}$ values from each line with sex-averaged genetic distances available for each chromosome [31]. Ne estimates from experimental populations were corrected for chromosomal sample size. In general, the $\mathrm{Ne}$ size was larger in BL than WL populations based on the comparison of two data sets and parallel SNP panels reflecting less inbreeding among brown, than white, egg layers (Figure 6). The recent (5 generations ago) and ancestral (200 generations ago) effective population size was estimated to be less than 70 and 300, respectively, for BL versus 50 and 100 individuals, respectively, for WL, displaying clear evidence of a decline in effective population size.

We have further delineated the influence of demography on LD by contrasting the differences observed between the WL and BL populations in different data sets. The commercial and experimental lines both revealed a clear evidence of decaying $\mathrm{Ne}$, however, the trend of decaying ancestral $\mathrm{Ne}$ appears to be stronger in the commercial populations than in the experimental lines. In contrast recent $\mathrm{Ne}$ for commercial lines is in general larger than for experimental lines, reflecting different constraints and breeding management in their

Table 4 Comparison of the strength of LD versus physical distance

\begin{tabular}{|c|c|c|c|c|c|c|c|c|}
\hline \multirow[b]{3}{*}{ Distance (Mb) } & \multicolumn{4}{|c|}{ Data set I } & \multicolumn{4}{|c|}{ Data set II } \\
\hline & \multicolumn{2}{|c|}{ BE } & \multicolumn{2}{|c|}{ WE } & \multicolumn{2}{|r|}{$\mathrm{BC}$} & \multicolumn{2}{|c|}{ WC } \\
\hline & Mean $\pm S D$ & Useful LD (\%) & Mean $\pm S D$ & Useful LD (\%) & Mean $\pm S D$ & Useful LD (\%) & Mean $\pm S D$ & Useful LD (\%) \\
\hline$<0.025$ & $0.37 \pm 0.34$ & 48.6 & $0.66 \pm 0.38$ & 76 & $0.32 \pm 0.33$ & 36.3 & $0.73 \pm 0.36$ & 79 \\
\hline $0.025-0.05$ & $0.32 \pm 0.32$ & 43.3 & $0.61 \pm 0.39$ & 73 & $0.28 \pm 0.30$ & 31.4 & $0.67 \pm 0.38$ & 74 \\
\hline $0.05-0.075$ & $0.29 \pm 0.30$ & 39.9 & $0.56 \pm 0.39$ & 68 & $0.24 \pm 0.28$ & 27.9 & $0.64 \pm 0.38$ & 72 \\
\hline $0.075-0.12$ & $0.27 \pm 0.29$ & 36.7 & $0.51 \pm 0.38$ & 64 & $0.21 \pm 0.26$ & 24.5 & $0.60 \pm 0.38$ & 68 \\
\hline $0.12-0.2$ & $0.24 \pm 0.26$ & 32.8 & $0.45 \pm 0.37$ & 59 & $0.18 \pm 0.23$ & 19.8 & $0.54 \pm 0.38$ & 63 \\
\hline $0.2-0.5$ & $0.19 \pm 0.23$ & 25.0 & $0.35 \pm 0.33$ & 47 & $0.13 \pm 0.18$ & 12.9 & $0.44 \pm 0.36$ & 52 \\
\hline $0.5-1.5$ & $0.12 \pm 0.17$ & 13.8 & $0.19 \pm 0.24$ & 25 & $0.07 \pm 0.12$ & 4.8 & $0.26 \pm 0.30$ & 31 \\
\hline $1.5-3$ & $0.07 \pm 0.12$ & 6.5 & $0.09 \pm 0.15$ & 10 & $0.04 \pm 0.07$ & 1.5 & $0.15 \pm 0.23$ & 16 \\
\hline $3-5$ & $0.05 \pm 0.08$ & 3.4 & $0.05 \pm 0.09$ & 04 & $0.02 \pm 0.04$ & 0.4 & $0.08 \pm 0.15$ & 6 \\
\hline $5-10$ & $0.04 \pm 0.06$ & 1.5 & $0.04 \pm 0.05$ & 01 & $0.01 \pm 0.02$ & 0.1 & $0.03 \pm 0.06$ & 1 \\
\hline
\end{tabular}

The mean $r^{2}$ values and the proportion of SNP pairs that shows statistically significant LD for markers apart up to 10 Mbp are presented for the all populations. 


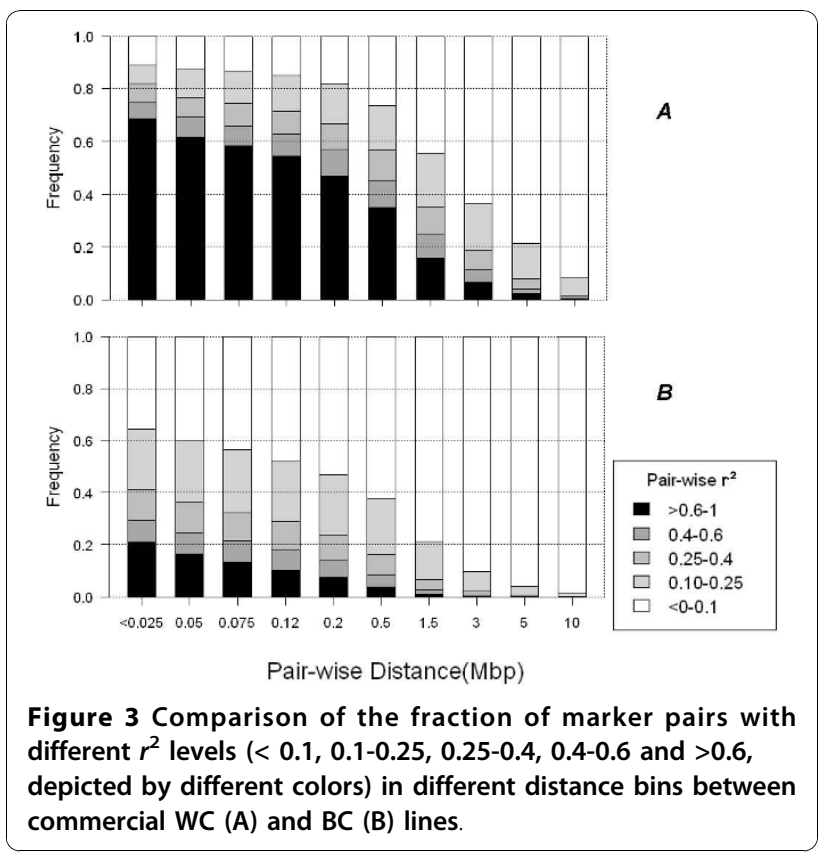

more recent history. The latter confirms the estimates of the current $\mathrm{Ne}$ based on information from pedigrees of experimental lines (R22, L68) in comparison to the Lohmann commercial lines of LSL-A and LB-A [37].

\section{Discussion}

In this article we report the construction of a wholegenome LD map using genotypes from white and brown egg laying chickens. We analyzed data from two parallel SNP chips comprising more than 40 '000 genetic markers, covering 28 chromosomes but leaving other microchromosomes uncovered because they had not

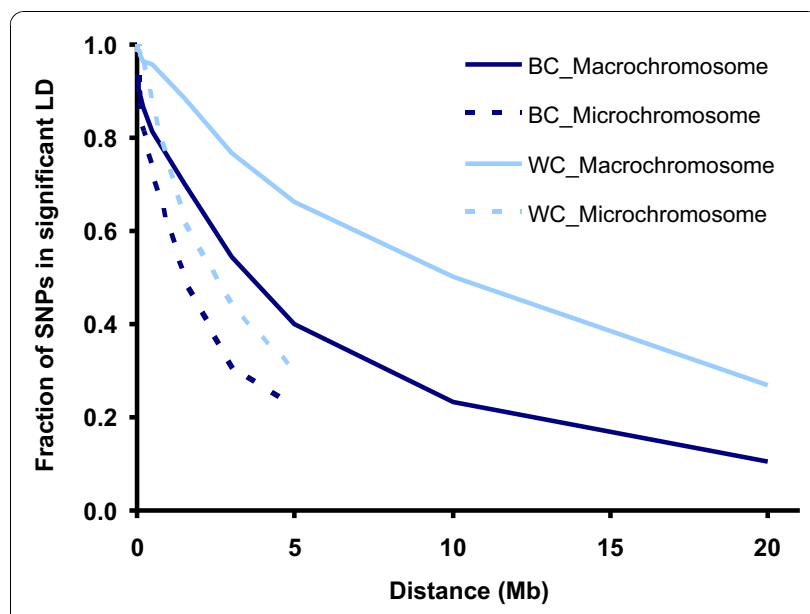

Figure 4 The proportion of SNP pairs in significant $(P \leq 0.05)$ LD plotted against the physical distance for macro- and microchromosomes in the commercial lines.

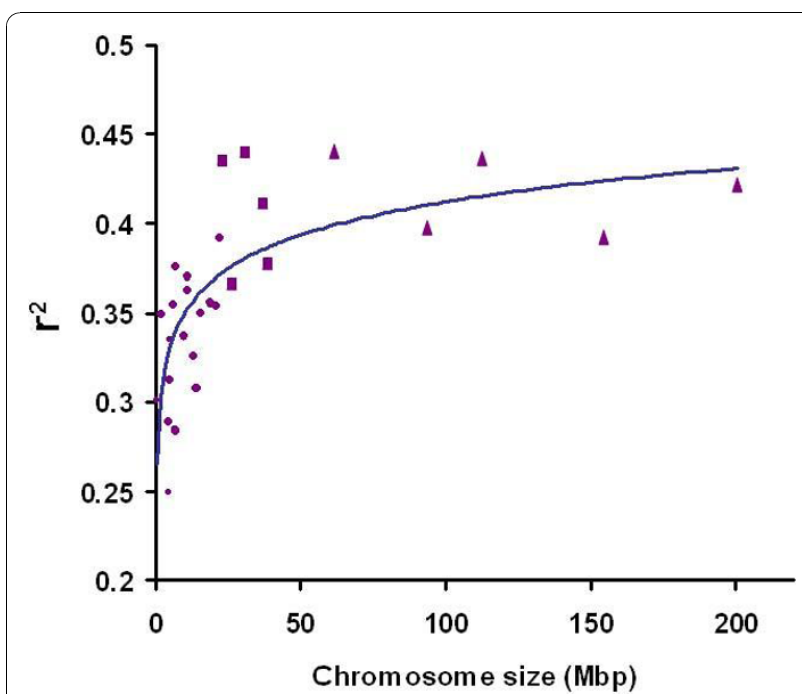

Figure 5 This plot depicts the relationship between the strength of LD versus length of the chromosomes in experimental Brown laying hens. Triangles, squares and circles are presenting mean $r^{2}$ across macro, intermediate and microchromosomes, respectively. The regression line was fitted as $y$ $=0.0269 \operatorname{Ln}(x)+0.2881$ with $R^{2}=0.52$.

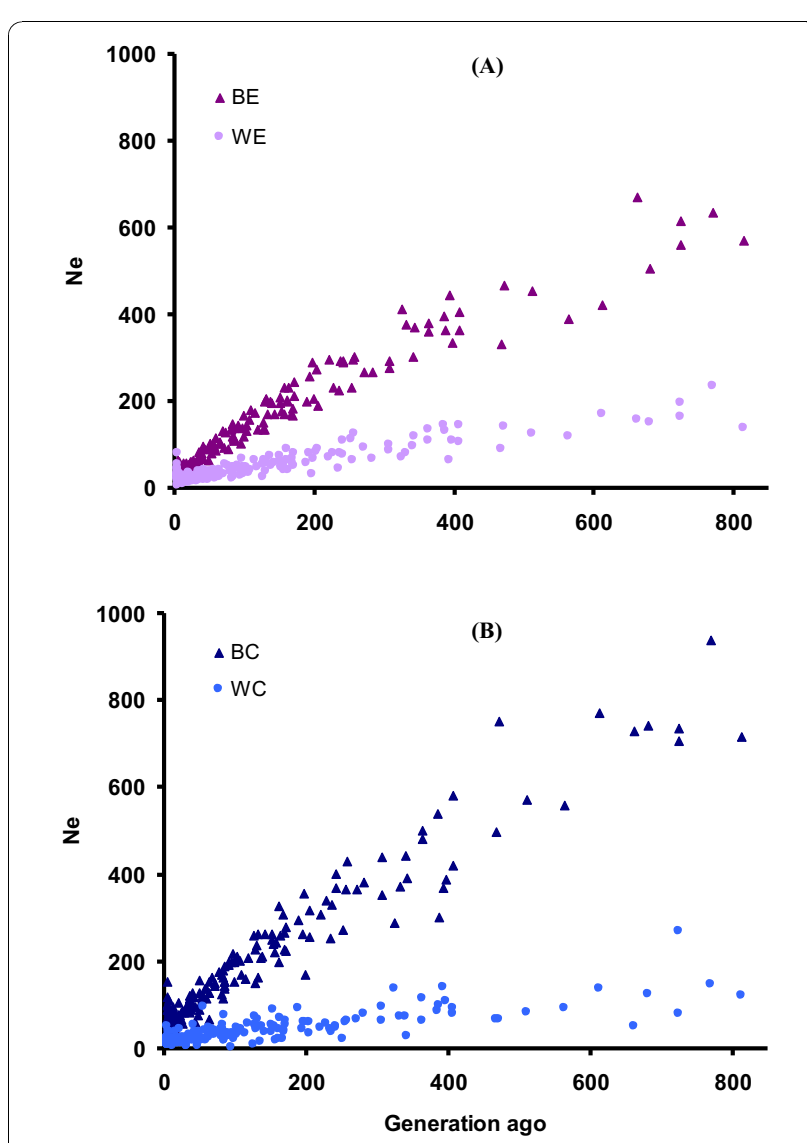

Figure 6 Decay in effective population size over generations for experimental (A) and commercial lines (B) estimated from linkage disequilibrium data. 


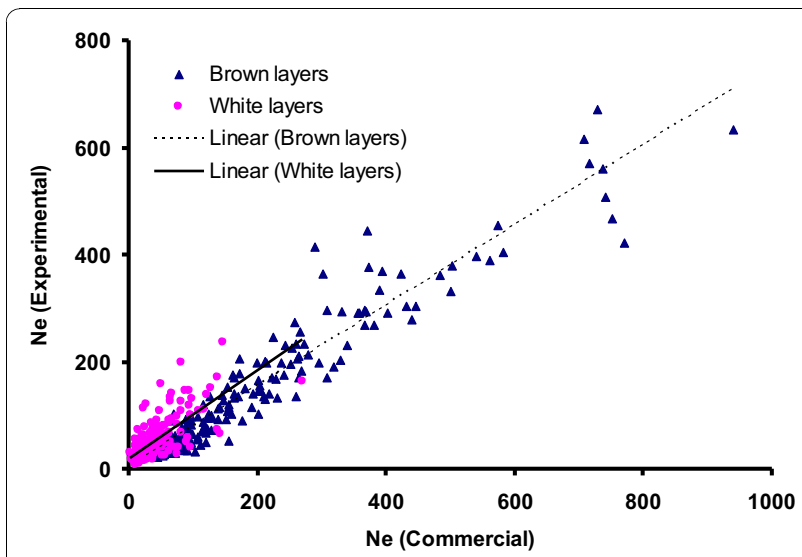

Figure 7 Estimates of $\mathrm{Ne}$ values from experimental white and brown layers are plotted against the commercial lines. $R^{2}$ of fitted lines were estimated as 0.92 and 0.57 for white and brown layers, respectively.

sufficiently large numbers of SNPs to obtain accurate results. This is so far the most comprehensive study of LD with high density SNP panels in breeding lines of egg laying chickens and currently reflects the best estimates of genome-wide LD based on the number of animals screened and number of SNPs genotyped.

We found a widespread LD among markers separated up to $10 \mathrm{Mb}$ apart. Although there were significant differences in the degree of LD between lines, overall LD levels were high. The magnitude of LD appeared to be greater and to extend over longer distances in WL compared to BL lines. This can be attributed to the very effective and intense long-term selection in the WL population. In contrast, the BL was originally a dual-purpose breed selected for multiple objectives and was subject to advanced selection procedures much later than WL. Furthermore, white egg layers originate from a single breed only, the single comb White Leghorn, while the genetic base of brown layers was broader [38]. A first study of the level of LD in chicken was conducted on a number of breeding populations of layer chickens [8]. In this study LD was evaluated using the standardized chi-square $\left(\chi^{2}\right)$ measure and showed appreciable LD among markers up to $5 \mathrm{cM}$ apart. This study, however, was based on microsatellites instead of SNPs and uses a different statistic compared to our study. Aerts et al. [33] investigated the extent of LD on the two chromosomes 10 and 28 in a white layer population and two broiler chicken breeds. The inbred layer breed showed an extent of useful LD up to $4 \mathrm{cM}$ on chromosome 10. They also found that the extent of LD varies dramatically. In a study of Andreescu et al. [9] genotype data for 959 and 398 SNPs on chromosomes 1 and 4 from nine commercial broiler chicken breeding lines were used. They reported that $r^{2}$ extends only over relatively short distances $(<1 \mathrm{cM})$. Further studies with $3 \mathrm{~K} \mathrm{SNP} \mathrm{data}$ suggested that in general the level of LD in WL $\left(r^{2}>0.2\right.$ at $<2 \mathrm{Mb}$ ) is higher than broilers [11]. While the $3 \mathrm{k}$ chip was not of sufficient density to reveal LD statistics, it was adequate for studying impacts of commercialization on allelic diversity and inbreeding. A study by Muir et al. [32] reported a higher level of inbreeding and lower allelic diversity in broilers than in egg laying chickens which is consistent with the results of this study.

In the current study, we aimed at dissecting the LD pattern more precisely by employing a higher marker density. Our results suggest that the level of LD in brown egg laying chickens is much less than previously assumed. However, the level of LD in the white egg layer lines we studied appears to be similar to the values reported with a lower marker density, which is consistent with the relatively small estimated effective population sizes of WL populations.

The decay of LD in a genome determines the resolution of quantitative trait loci detection in association mapping studies and indicates the required marker density. The substantial differences in the LD pattern between lines reflected by different relationship between $r^{2}$ and the physical chromosome length (Figure 4) confirms the complex nature of LD in chicken. This has consequences for QTL mapping and, in particular, fine mapping studies. LD in macrochromosomes extends over large genomic regions so that there is a higher chance of finding association between a gene affecting a particular phenotype and a marker at a given distance. This means that a modest number of markers should be sufficient to identify macrochromosomal QTL by association mapping. However, with long-range LD, the physical distance between a gene and an associated marker can be substantial, making fine mapping and gene identification more challenging. Conversely, on microchromosomes, the fast decay of LD over short distances requires a much denser marker map for finding associations. However, when found, gene annotation and identification will be less tedious [39].

For genomes of mammalian farm animals (e.g. cattle) with an approximate average ratio of $1 \mathrm{Mbp} / 1$ $\mathrm{cM}$, the required level of $\mathrm{LD}\left(r^{2}\right)$ for genomic association studies was suggested to be in range of 0.2-0.3 $[34,23]$. Considering that the linkage distance in chicken is on average 3 times larger than in mammals, current results indicate that the SNP spacing should be $\sim 100 \mathrm{~Kb}$ and $\sim 35 \mathrm{~Kb}$, respectively, for commercial white and brown layers to achieve a comparable coverage in future population wide studies with a whole-genome approach. This implies that the current density of informative SNPs used may be adequate, provided that SNPs are distributed across the microand macrochromosomes proportionally to their recombination rates. 
The extent of haploblock structures in commercial populations of chicken is not well enough documented to allow a comparison with the results of the current study. Most recently, Megens et al. [40] reported haploblock length of less than $10 \mathrm{~kb}$ in the genome of some commercial lines. However, the study was limited to targeted regions of only two macro and two micro-chromosomes covered by SNP sets of high density. In contrast to the haploblock structure in other species, average block size observed in the present study is comparable to the one recently observed in cattle [17] and at the same time is 20-30 times larger than the one reported in human genomes [41]. It must be noted that the marker density used in this study is more than 50 times sparser than the one currently being used for the human genome. In cattle only a minor part (i.e., 4\%) of the genome could be assigned to haplotype blocks, indicating that the SNP density was not sufficient. With a denser bovine SNP panel, shorter blocks will be identifiable and the average haploblock size is expected to decrease. In the current study, the haploblocks account for a substantial proportion of the entire genome coverage (especially so in the white layers), hence a denser SNP panel will not lead to the identification of large numbers of short blocks, but in agreement with the observations by Megens et al. [40] the large blocks found with a coarse SNP panel may possibly split up in series of small blocks with a denser panel.

In typical livestock breeding populations, where intensive selective breeding is practiced, effective population size plays a central role because it affects both the degree to which a population can respond to selection and its sensitivity to inbreeding effects [42-44]. Given these implications, the knowledge of $N_{\mathrm{e}}$ facilitates the design of efficient artificial selection schemes [45]. As highlighted by Wang [46] $\mathrm{Ne}$ is notoriously difficult to estimate, mainly due to the highly stochastic nature of the processes underlying inbreeding and genetic drift.

We estimated historical $\mathrm{Ne}$ based on information from LD for each population separately. The results were found to be consistent between analyses based on the parallel SNP chips, indicating that results were independent of the panel used, as expected when SNPs are neutral and most LD is generated by drift (Figure 7).

The effective population sizes that were estimated in experimental lines are generally in agreement with current estimates of $\mathrm{Ne}$ based on information from pedigrees of these populations [37]. Our estimates of $\mathrm{Ne}$ in layer lines are substantially smaller than those obtained for the broiler lines reported by Andreescu et al. [9], which confirms the higher estimates for broiler than for layer lines observed by Muir et al. [32]. Indeed, differences in $\mathrm{Ne}$ between WL and BL lines are consistent with the breed histories of those lines.

\section{Conclusions}

This is a comprehensive study of LD based on high density SNP panels in breeding lines of egg laying chickens and currently reflects the best estimates of genome-wide LD based on the number of animals screened and number of SNPs genotyped. The number of usable SNPs in this study was $19 \mathrm{k}$ to $37 \mathrm{k}$ in brown layers and $8 \mathrm{k}$ to $19 \mathrm{k}$ in white layers. The results were found to be consistent between analyses based on the parallel SNP chips and across different types of populations (commercial vs. experimental) within the brown and the white layers, respectively. We found that LD generally decays rapidly with distance in different lines, but there was substantial variation between WL versus $\mathrm{BL}$ and subtle difference between commercial and experimental populations. If the pattern observed in this study holds true for most of the genome, it is indicated that the current density of usable markers in this study is sufficient for association mapping and implementation of genomic selection within these populations to achieve a comparable resolution and accuracy as in mammalian farm animal populations with applications of 50 to $60 \mathrm{k} \mathrm{SNP}$ chips. If genomic predictions are to be made across lines, higher SNP densities may be advantageous.

\section{List of abbreviations}

LD: Linkage disequilibrium; Ne: effective population size; WC: white commercial; WE: white experimental; BC: brown commercial and BE: brown experimental

\section{Authors' contributions}

SQ performed the statistical analyses and wrote the final draft and prepared the manuscript for submission. HS supervised the study and contributed in the conception of the study and revising and editing the manuscript. $\mathrm{MH}$ collaborated in generation of data. SW participated in provision of data and in reviewing the manuscript for scientific content. RP participated in provision of study material and manuscript improvement and also provided administrative support. All authors read and approved the manuscript.

\section{Acknowledgements}

This research was funded by the German Federal Ministry of Education and Research (BMBF) within the AgroClustEr "Synbreed - Synergistic plant and animal breeding" (FKZ: 0315528A). Parts of the data used were generated within the project FUGATO E.coli - chick which was financially supported by BMBF and Lohmann Tierzucht GmbH, Cuxhaven. MH, SW and HS thank the German Research Foundation (DFG; SI 381/11-1 and WE 1901/3 - 1) for financial support. We thank Mrs. Anke Flörke for preparing the DNA samples and Mrs. Ute Döring for support in language editing.

\section{Author details}

${ }^{1}$ Animal Breeding and Genetics Group, Department of Animal Sciences, Georg-August University, 37075 Göttingen, Germany. ${ }^{2}$ Institute of Farm Animal Genetics, Friedrich-Loeffler-Institut, Neustadt-Mariensee, Germany. ${ }^{3}$ Lohmann Tierzucht GmbH, Cuxhaven, Germany.

Received: 11 August 2010 Accepted: 15 November 2010 Published: 15 November 2010

\section{References}

1. Van Laere AS, Nguyen M, Braunschweig M, Nezer C, Collette C, Moreau L, Archibald AL, Haley CS, Buys N, Tally M, Andersson G, Georges M, 
Andersson L: A regulatory mutation in IGF2 causes a major QTL effect on muscle growth in the pig. Nature 2003, 425:832-836.

2. Grisart B, Farnir F, Karim L, et al: Genetic and functional confirmation of the causality of the DGAT1 K232A quantitative trait nucleotide in affecting milk yield and composition. Proc Nat Aca Sci 2004, 101:2398-2403.

3. Dekkers JCM: Commercial application of marker- and gene-assisted selection in livestock: strategies and lessons. J Anim Sci 2004, 82(13 Electronic Supplement):E313-E318.

4. Sved JA: Linkage disequilibrium and homozygosity of chromosome segments in finite populations. Theor Popul Biol 1971, 2(2):125-141.

5. Serebrovskay S, G Petrov S: A case of close autosomal linkage in the fowl. $J$ Hered 1930, 19:305-306.

6. Cheng HH, Levin I, Vallejo RL, H K, Dodgson JB, Crittenden L, Hillel J: Development of a genetic map of the chicken with markers of high utility. Poult Sci 1995, 74:1855-1874, 1930 On the composition of the plan of the chromosomes of the domestic fowl. J. Exper. Biol. (Russian) 6: 157-179. (From translation by B. F. Glessing. U. S. Dept. Agric.).

7. Groenen MA, Cheng H, Bumstead N, Benkel B, Briles W, Burke T, Burt DW, Crittenden L, Dodgson JB, Hillel J, et al: A Consensus Linkage Map of the Chicken Genome. Genome Res 2000, 10:137-147.

8. Heifetz EM, Fulton JE, O'Sullivan N, Zhao H, Dekkers JC, Soller M: Extent and consistency across generations of linkage disequilibrium in commercial layer chicken breeding populations. Genetics 2005, 171(3):1173-1181.

9. Andreescu C, Avendano S, Brown SR, Hassen A, Lamont SJ, Dekkers JC: Linkage disequilibrium in related breeding lines of chickens. Genetics 2007, 177(4):2161-2169.

10. Groenen MA, Wahlberg P, Foglio M, Cheng $H$, Megens $H_{\text {, }}$ Crooijmans RPMA, Besnier F, Lathrop GM, Muir WM, Wong GKS, et al: A high-density SNP-based linkage map of the chicken genome reveals sequence features correlated with recombination rate. Genome Res 2009, 19:510-519.

11. Abasht B, Sandford E, Arango J, Settar P, Fulton JE, O'Sullivan NP, Hassen A, Habier D, Fernando RL, Dekkers JC, Lamont SJ: Extent and consistency of linkage disequilibrium and identification of DNA markers for production and egg quality traits in commercial layer chicken populations. BMC Genomics 2009, 14(10 Suppl 2):S2.

12. Elferink MG, van As P, Veenendaal T, Crooijmans RP, Groenen MA: Regional differences in recombination hotspots between two chicken populations. BMC Genetics 2010, 8(11):11.

13. Consortium ICPM: A genetic variation map for chicken with 2.8 million single-nucleotide polymorphisms. Nature 2004, 432(7018):717-722.

14. Hartmann W: Evaluation of major genes affecting resistance to disease in poultry. Wor Poul Sci J 1997, 53(3):231-252.

15. Sambrook J, Russell DW: Molecular Cloning: A Laboratory Manual. Cold Spring Harbor Laboratory, New York; 2001.

16. Lewontin RC: The detection of linkage disequilibrium in molecular sequence data. Genetics 1995, 140:377-388.

17. Qanbari S, Pimentel ECG, Tetens J, Thaller G, Lichtner P, Sharifi AR, Simianer $\mathrm{H}$ : The pattern of linkage disequilbriom in German Holstein cattle. Animal Genetics 2010, 41:346-356.

18. Bonnen PE, Wang PJ, Kimmel M, Chakraborty R, Nelson DL: Haplotype and linkage disequilibrium architecture for human cancer-associated genes. Genome Res 2002, 12:1846-1853.

19. Scheet P, Stephens M: A fast and flexible statistical model for large-scale population genotype data: Applications to inferring missing genotypes and haplotypic phase. Am J Hum Genet 2006, 78:629-644.

20. Barrett JC, Fry B, Maller J, Daly MJ: Haploview: analysis and visualization of LD and haplotype maps. Bioinformatics 2005, 21(2):263-265.

21. Gabriel SB, Schaffner SF, Nguyen H, Moore JM, Roy J, Blumenstiel B, Higgins J, DeFelice M, Lochner A, Faggart M, Liu-Cordero SN, Rotimi C, Adeyemo A, Cooper R, Ward R, Lander ES, Daly MJ, Altshuler D: The structure of haplotype blocks in the human genome. Science 2002, 296:2225-2229.

22. Kruglyak L: Prospects for whole-genome linkage disequilibrium mapping of common disease genes. Nat Genet 1999, 22:139-144.

23. Ardlie KG, Kruglyak L, Seielstad M: Patterns of linkage disequilibrium in the human genome. Nat Rev Genet 2002, 3:299-309.

24. Terwilliger JD, Weiss KM: Linkage disequilibrium mapping of complex disease: fantasy or reality? Cur Opin Biot 1998, 9:578-594.
25. Hill WG, Weir BS: Maximum-likelihood estimation of gene location by linkage disequilibrium. Am J Hum Genet 1994, 54:705-714.

26. Hill AP: Quantitative linkage: a statistical procedure for its detection and estimation. Ann Hum Genet 1975, 38:439-449.

27. Hayes BJ, Visscher PM, McPartlan HC, Goddard ME: Novel multilocus measure of linkage disequilibrium to estimate past effective population size. Genome Res 2003, 13:635-643.

28. Tenesa A, Knott SA, Ward D, Smith D, Williams JL, et al: Estimation of linkage disequilibrium in a sample of the United Kingdom dairy cattle population using unphased genotypes. J Anim Sci 2003, 81:617-623.

29. The International Human Genome Sequencing Consortium: Initial sequencing and analysis of the human genome. Nature 2001, 409:860-921.

30. Mouse Genome Sequencing Consortium: Initial sequencing and comparative analysis of the mouse genome. Nature 2002, 420:520-562

31. Consortium ICGS: Sequence and comparative analysis of the chicken genome provide unique perspectives on vertebrate evolution. Nature 2004, 432:695-716

32. Muir WM, et al: Genome-wide assessment of worldwide chicken SNP genetic diversity indicates significant absence of rare alleles in commercial breeds. Proc Nat Aca Sci 2008, 105(45):17312-17.

33. Aerts J, Megens HJ, Veenendaal T, Ovcharenko I, Crooijmans R, Gordon L, Stubbs L, Groenen M: Extent of linkage disequilibrium in chicken. Cytogenet Genome Res 2007, 117(1-4):338-345.

34. Meuwissen TH, Hayes BJ, Goddard ME: Prediction of total genetic value using genome-wide dense marker maps. Genetics 2001, 157(4):1819-1829.

35. McQueen HA, Siriaco G, Bird AP: Chicken microchromosomes are hyperacetylated, early replicating, and gene rich. Genome Research 1998, 8:621-630.

36. Axelsson $\mathrm{E}$, et al: Comparison of the chicken and turkey genomes reveals a higher rate of nucleotide divergence on microchromosomes than macrochromosomes. Genome Research 2005, 15:120-125.

37. Hansen M, Weigend S, Simianer H: Estimation Of Effective Population Size In Chicken Breeding Based On Chromosome Segment Homozygosity. The 23rd World's Poultry Congress, Brisbane, 10 - 15 August 2008, Australia

38. Crawford RD: Origin and history of poultry species. In Poultry Breeding and Genetics. Edited by: Crawford RD. Elsevier Amsterdam, The Netherlands; 1990:1-42.

39. Backström N, Qvarnström A, Gustafsson L, Ellegren H: Levels of linkage disequilibrium in a wild bird population. Biol Lett 2006, 2:435-438.

40. Megens HJ, Crooijmans RPMA, Bastiaansen JWM, Kerstens HHD, Coster A, Jalving R, Vereijken A, Silva P, Muir WM, Cheng HH, Hanotte O, Groenen MAM: Comparison of linkage disequilibrium and haplotype diversity on macro- and microchromosomes in chicken. BMC Genetics 2009, 10:86.

41. Hinds DA, Stuve LL, Nilsen GB, Halperin E, Eskin E, Ballinger DG, Frazer KA, Cox DR: Whole-genome patterns of common DNA variation in three human populations. Science 2005, 307:1072-1079.

42. Crow JF, Kimura M: An Introduction to Population Genetics Theory Harper \& Row, New York; 1970.

43. Lande R: Mutation and conservation. Conserv Biol 1995, 9:782-791.

44. Lynch M, Conery J, Burger R: Mutational meltdowns in sexual populations. Evolution 1995, 49:1067-1080

45. Caballero A, Keightley PD, Hill WG: Strategies for increasing fixation probabilities of recessive mutations. Genet Res 1991, 58:129-138.

46. Wang J: Estimation of effective population sizes from data on genetic markers. Philos Trans R Soc Lond B Biol Sci 2005, 360(459):1395-409.

doi:10.1186/1471-2156-11-103

Cite this article as: Qanbari et al: Linkage disequilibrium reveals different demographic history in egg laying chickens. BMC Genetics 2010 11:103. 